\title{
Special issue. Aboriginal voices: Systematic reviews of indigenous education
}

\author{
John Guenther $^{1} \cdot$ Neil Harrison ${ }^{2}$ (D) . Cathie Burgess ${ }^{3}$
}

Received: 19 February 2019 / Accepted: 26 February 2019 / Published online: 22 March 2019

(c) The Australian Association for Research in Education, Inc. 2019

This most ambitious series of systematic reviews of the literature (SRL) associated with the field of Aboriginal and Torres Strait Islander school education brings together nine academics from six Australian higher education institutions, to review over 10,000 papers.

The SRL arose from the recognition that Aboriginal and Torres Strait Islander school education in Australia has become mired in myths and legends. Despite the millions of dollars that are spent on 'new' programs, and despite the best intentions of researchers over the previous 15-20 years to identify the ingredients for success, there was, on the surface of things, little evidence to support the claims. We considered that it was time to re-evaluate the field and its achievements. The SRL was thus born out of a combined desire amongst experienced researchers across Australia to differentiate fact from fiction, and to identify, through careful analysis, the future goals and directions of Indigenous education.

It became clear through our systematic reviews that there is a strong need for a national vision. The states and territories work in their silos, and education is seen mainly as a pursuit for individuals. The work presented here is the result of academics with specialised interests in Aboriginal and Torres Strait Islander education, working together over 2 years to better understand the research evidence. The papers in this collection indeed reflect this close collaboration. The team has worked together to write and edit each of the papers presented in this collection. Hence, multiple members of the SRL team are recognised as authors on each of the papers. It is an unprecedented process and indeed reflects what could contribute to a national vision for Australia, of Aboriginal and Torres Strait Islanders and the non-Indigenous Australians working together to decolonise the parochial concepts of targets

Neil Harrison

neil.harrison@mq.edu.au

1 Batchelor Institute of Indigenous Tertiary Education, Cnr Nurndina St and Kirra Cresc, Batchelor, NT 0845, Australia

2 Macquarie University, Sydney, NSW 2109, Australia

3 University of Sydney, Camperdown, NSW 2006, Australia 
and outcomes that currently shackle the works of teachers, students, their parents and community in the field of Aboriginal and Torres Strait Islander education.

In the context of looming adjustments to the Federal Government's Closing the Gap policy, the team presented in 2018 to the Department of Prime Minister and Cabinet, and to the Deputy Secretary of Indigenous Affairs, Professor Ian Anderson. This followed presentations at the Australian Association for Research in Education (AARE) in 2017, and again in 2018.

\section{Seven papers presented in this special edition}

The first paper by Lowe, Tennent, Guenther, Harrison, Burgess, Moodie and Vass describes the methodological approach adopted for the SRL, and for each of the other papers. The Review initially established an agreed common set of protocols which mapped the primary question/s for each review, the search strategies to implement, the criteria for the inclusion and exclusion of studies and a robust way of assessing the research quality of these studies. The methodology paper highlights how the legitimacy of a SRL, as a research methodology is based on the thoroughness of the process, the rigour of its internal protocols and methodical way these are implemented.

The second paper by Harrison, Tennent, Vass, Guenther, Lowe and Moodie explores the question of how the curriculum governs learning and teaching in Aboriginal and Torres Strait Islander education. This paper calls into question the purpose and goals of education and argues that the government's current focus on numbers contrasts radically with the ways in which many Aboriginal and Torres Strait Islander students and their parents conceptualise these fundamental principles. Moreover, the research analysed demonstrates that what counts as knowledge in the Australian Curriculum also contrasts with Aboriginal and Torres Strait Islander views which, in various forms, identify Country as an enactment of curriculum and therefore challenges normalised discourses about the very essence of curriculum.

The third paper from Lowe, Harrison, Tennent, Guenther, Vass and Moodie focuses on community engagement, and the factors affecting the development of school and Aboriginal community engagement. This review provides insights into the everyday environments of interactions between schools and Aboriginal families and communities, the possibilities that emanate from the establishment of collaborations between these groups and the hope that these interactions can positively impact students' educational outcomes. The issues that emerged from this analysis are complex, bounded by the uniqueness of particular sites including their histories, economies and location, their interactions with schools and their experiences of educational success and engagement.

The fourth paper from Moodie, Maxwell and Rudolph investigates racism in education. This review attends to diverse manifestations of racism, from institutional and systemic discrimination to everyday microaggressions. Empirical evidence demonstrates that racism negatively impacts experiences of Aboriginal and Torres Strait Islander students, and that these impacts are harmful, wide-reaching and life-long. They influence academic achievement, attitudes to language, emotional 
wellbeing, physical health, self-concept, school attendance, post-school pathways, and eventually school choice and parental school engagement.

The fifth paper by Burgess, Tennent, Vass, Guenther, Lowe and Moodie analyses those pedagogies that have been demonstrated to support, engage and improve the educational outcomes of Aboriginal students. Whilst many studies identified successful teaching practices for engaging and supporting Aboriginal students, this SLR was not able to locate any research conducted since 2006 that demonstrates impact on learning outcomes. The evidence is simply not there to show what successful pedagogy actually looks like in Indigenous contexts. Further, the role of context (mainly located in remote areas) inadvertently illuminated the paucity of research in urban locations and highlighted an enduring dilemma of how to balance the significance of local context with identifying effective pedagogic frameworks that can be applied more broadly to improve Aboriginal student outcomes.

The sixth, from Guenther, Lowe, Burgess, Vass and Moodie investigates the factors that contribute to educational outcomes for Aboriginal and Torres Strait Islander students from remote communities. This review raises important questions about whose conceptualisation of outcomes matter, those within the hegemonic power structures that define education policy which are configured around literacy, numeracy, retention, transition to higher education and transition to jobs, or those defined from a community perspective such as parent and community involvement, attention to health, safety and wellbeing, local employment, appropriate curriculum and pedagogies and strategies that build engagement in learning. Guenther et al. also flag the complexity of context and expose commonly held myths such as remoteness or disadvantage itself as a problem to be overcome. They further identify key gaps in the literature pertaining to leadership, funding, policy, workforce development and pre-service teacher preparation.

The final paper from Vass, Lowe, Burgess, Harrison and Moodie analyses what professional learning has been found to support Indigenous students in schools. The themes emerging from this review emphasise the importance of finding ways to more genuinely ensure that Indigenous peoples contribute to leading teacher professional learning that also explicitly addresses issues related to culture, (anti) racism, power and relationships in schooling. The SLR found that when Indigenous epistemologies and methodologies are embedded in classroom learning through the genuine involvement of Aboriginal and Torres Strait Islander researchers, learning outcomes for both teachers and their students improved.

Not surprisingly given the diversity of Aboriginal and Torres Strait Islander communities across Australia, the role of context emerged as a strong theme across the reviews, highlighting the complexity of meeting the needs of specific communities while identifying effective strategies that can be applied more broadly. It also illuminated the paucity of research in urban locations, thus foregrounding the invisibility of urban students, their families, communities and the specific issues that affect their education.

Significantly, fundamental issues such the goal and purpose of education, how outcomes are defined and by whom, and what success might look like from an Indigenous perspective all emerged as key contested sites. Certainly, the need to 
re-define success from Indigenous standpoints beyond short-term quantifiable targets such as external test scores was flagged in a number of research studies.

The aggregation of the individual review findings enabled the identification of the core interconnected themes of Indigenous identity and connectedness. These themes particularly focused on the importance of relationality between students and their communities, to their Country and its knowledge and the importance of family and community engagement to effective teaching and curriculum. The crucial importance of student access to language and cultural programmes was also flagged in a number of studies as being crucial to Aboriginal student engagement in their education.

A disturbing finding from the systematic reviews noted the failure to identify any one pedagogic approach that significantly impacted on the engagement and outcomes of Indigenous students or any established professional development programmes that saw a significant shift in teachers' general and/or pedagogic practices. This point was taken up by Harrison et al. who identified the impact of 'place-based' curriculum in accommodating the 'competing' knowledge systems of the state (as represented within the mandated curriculum) and Indigenous epistemologies within the classroom. Recognising that this is rarely seen within Australian classrooms is not surprising, with Vass et al. and Burgess et al. noting the paucity of Australian research on schools' capacity to shift teachers' classroom practice, and building meaningfully engagement with students' families in support of their education.

The SRL highlights how the detrimental effects of racism on Indigenous student identities, self-esteem and success at school continues to be pervasiveness. Indigenous realities of colonisation, racism, and underachievement, highlight the presence of deeply embedded racist practices in schools, and must be of grave concern to all.

The range of issues uncovered in the SRL reflects the complexity of the field. There is no silver bullet solution that will 'fix' education for Aboriginal and Torres Strait Islander students. However, the array of findings emerging from papers suggests a need to ensure that priorities of students, communities and parents are brought to the fore in the development of strategies that must be implemented and applied at the level of local communities. The days of one single federal policy designed to meet all needs and situations appears to be at an end. Coupled with this, we see the importance of culture and language consistently emerging, as non-negotiable for contextually responsive pedagogies.

Further, despite the thousands of papers reviewed, there continues to be a dearth of empirical evidence to support policy and good practice. We know many of the stories and experiences of students and teachers who are so often working hard to make things work. But we are yet to measure the impact of these approaches on educational outcomes. And we still do not know how the various Indigenous communities throughout Australia; urban, rural and remote measure these learning outcomes in their own terms.

Whilst this project has reviewed past research conducted in the field of Indigenous school education, we have been driven by a vision for Australia that is based on national recognition of its colonial past and a desire for co-existence in the future. 
Publisher's Note Springer Nature remains neutral with regard to jurisdictional claims in published maps and institutional affiliations.

Prof John Guenther is a researcher with 20 years' experience working in overlapping fields of social inquiry, typically in areas related to education and training, and its intersections with mental health and wellbeing, justice, employment, child protection and welfare. In the last 10 years he has more intentionally focussed on issues affecting Aboriginal and Torres Strait Islander people living in remote parts of Australia. He led the Remote Education Systems project under the CRC for Remote Economic Participation (2011-2016) and is currently the Research Leader for Education and Training with the Batchelor Institute of Indigenous Tertiary Education, based in Darwin, Northern Territory. John is a leading academic in the field of remote education and has published widely on his findings, often under the banner of 'Red Dirt Thinking'.

Prof Neil Harrison research investigates ways in which Indigenous knowledge of Country can be embedded in the Australian Curriculum. His latest book (together with Juanita Sellwood), Learning and Teaching in Aboriginal and Torres Strait Islander Education (Oxford) is used widely in teacher education programs throughout Australia. He is currently developing an approach for teaching about the experiences of trauma in universities and schools.

Dr Cathie Burgess coordinates Aboriginal Studies, Aboriginal Community Engagement and the Master of Education: Leadership in Aboriginal Education programs. Cathie's research involves communityled initiatives positioning Aboriginal cultural educators as experts through projects such as Learning from Country in the City, Aboriginal Voices: Insights into Aboriginal Education and Redfern Cultural Program. 